\title{
Radio Propagation Measurements and Cluster-Based Analysis for 5G Millimeter-Wave Cellular Systems in Dense Urban Environments
}

This paper was downloaded from TechRxiv (https://www.techrxiv.org).

\section{LICENSE}

CC BY 4.0

\section{SUBMISSION DATE / POSTED DATE}

$21-07-2020$ / 23-07-2020

\section{CITATION}

Zhang, Peize; Yang, Bensheng; Wang, Haiming; Wang, Cheng-Xiang; You, Xiaohu (2020): Radio Propagation Measurements and Cluster-Based Analysis for $5 \mathrm{G}$ Millimeter-Wave Cellular Systems in Dense Urban Environments. TechRxiv. Preprint. https://doi.org/10.36227/techrxiv.12682049.v1

$\mathrm{DOI}$ 


\title{
Radio Propagation Measurements and
}

\section{Cluster-Based Analysis for 5G Millimeter-Wave}

\section{Cellular Systems in Dense Urban Environments}

\author{
Peize Zhang, Student Member, IEEE, Bensheng Yang, Student Member, IEEE, \\ Haiming Wang, Member, IEEE, Cheng-Xiang Wang, Fellow, IEEE, \\ and Xiaohu You, Fellow, IEEE
}

\begin{abstract}
Empirical channel modeling is necessary for the deployment of the fifth-generation (5G) millimeterwave (mmWave) cellular system in actual environments. In this paper, cluster-based analyses of mmWave channel characteristics in two typical dense urban environments are performed. First, radio propagation
\end{abstract}

Manuscript received March 1, 2020; revised July 9, 2020; accepted

Date of publication

; date of current

version . This work was supported in part by the National Key R\&D Program of China under Grant 2018YFB1801101, the National Natural Science Foundation of China under Grants 61960206006 and 61671145, and the Key R\&D Program of Jiangsu Province of China under Grant BE2018121. This paper was presented in part at the IEEE International Conference on Communications 2019 [1]. (Corresponding author: Haiming Wang.)

P. Z. Zhang, B. S. Yang, and H. M. Wang are with the State Key Laboratory of Millimeter Waves, Southeast University, Nanjing 210096, China and also with Purple Mountain Laboratories, Nanjing 211111, China (e-mail: pzzhang@seu.edu.cn, yangbensheng@seu.edu.cn, hmwang@seu.edu.cn).

C.-X. Wang and X. H. You are with the National Mobile Communications Research Laboratory, Southeast University, Nanjing 210096, China and also with Purple Mountain Laboratories, Nanjing 211111, China (e-mail: chxwang@seu.edu.cn, xhyu@seu.edu.cn). 
measurement campaigns are conducted at two primary $5 \mathrm{G}$ bands of $28 \mathrm{GHz}$ and $39 \mathrm{GHz}$ in a central business district and a dense residential area. The custom-designed channel sounder supports highefficiency directional scanning sounding, which helps to collect sufficient data for statistical channel modeling. Next, using an improved autoclustering algorithm, multipath clusters and their scattering sources are identified. Mapping results show that multiple reflections from exterior walls and diffraction over building corners or rooftops enhance the coverage for non-line-of-sight (NLoS) links and the influences of these propagation mechanisms are intuitively embodied as changes in the topologies of deployment environments. Finally, an appropriate measure for cluster-level channel characteristics is provided including cluster number, Ricean K-factor, root mean squared (RMS) delay spread, RMS angular spread, and their correlations. Comparisons of these parameters across two mmWave bands are also given. The measurement and modeling results shed light on a fully understanding of mmWave channels in dense urban environments across multiple bands.

\section{Index Terms}

Millimeter wave communication, propagation, multipath channels

\section{INTRODUCTION}

Millimeter-Wave (mmWave) communication, which makes use of abundant spectrum compared with the most commercial wireless systems operating below $6 \mathrm{GHz}$, has emerged as a key enabling technology to meet multi-gigabit-per-second data rate and millisecond level end-to-end latency for the fifth-generation (5G) mobile communication systems [2], [3]. Much as mmWave has been extensively used in IEEE 802.15.3c [4] and IEEE 802.11ad/aj [5], [6] standards to support local area short-range communication, the implementation of mmWave cellular networks in outdoor environments is much more challenging, where the aim is to provide feasible uplink and downlink transmission strategies, simplified network architectures, and cost-effective network deployments.

With the increase of carrier frequency, a distinguishing feature of outdoor cellular systems is the decrease of cell radii because of the large propagation attenuation and severe vulnerability 
to blockages. To achieve better outdoor coverage probability and enhance the connectivity, base station (BS) cooperation in mmWave ultra-dense networks is of great importance [7]. In addition, heterogeneous network integrating microwave macro BSs and mmWave small cell BSs is recognized to be a promising technology for 5G mmWave communications [7], [8]. The complicated network architectures indicate that traditional cellular networks with BSs on a grid are highly idealized and intractable for mmWave systems, which involve challenges with respect to deployment cost, power consumption, and coverage [9]. Hence, recently, there is great interest in theoretically and experimentally analyzing coverage and data rate of mmWave cellular networks from the perspective of realistic channel characteristics [10]-[12]. Another distinct feature of mmWave cellular communication is directional transmission. The sparse nature of mmWave propagation environments indicates that cluster-based representation of mmWave channel is reasonable, where the subpaths in each cluster share similar propagation delay and angle of arrival and departure [13], [14]. As a consequence, directional beams can be used to sweep through all possible directions and track effective clusters based on hybrid digital and analog beamforming [15], [16]. Thanks to the smaller wavelength at mmWave bands, the advent of large-scale antenna arrays with substantial array gain at transceivers will compensate excessive path loss for satisfactory coverage, while antenna aperture will not significantly increase [2].

Lately, extensive mmWave channel measurement campaigns have been conducted in various urban environments, such as university campus at 38, 60, and $73 \mathrm{GHz}$ [17], [18], densely built-up downtown at $28 \mathrm{GHz}$ and $60 \mathrm{GHz}$ [14], [19], street canyon at 28, 38, and $60 \mathrm{GHz}$ [13], [20]. The measurements show that an optimistic assessment of outdoor mmWave cellular systems can be performed with cell radii up to the order of $200 \mathrm{~m}$. Basically, highly directional horn antennas were leveraged to increase dynamic range of channel sounders and synthesize narrow beams for omnidirectional channel modeling via antenna steering in azimuth and elevation directions mechanically. It is worthy to mention that more advanced phased array based channel sounders have been developed for dynamic directional measurements [21]; however, it is acceptable that we 
still use directional scanning sounding (DSS) method for static channel measurements considering implementation cost. Meanwhile, there is a dearth of elaborate analysis on space-time channel characteristics down to the cluster level in existing studies. Only signals from one or two fixed zenith angles of arrival and departure $(\mathrm{ZoA} / \mathrm{ZoD})$ were measured due to the fact that traditional step-by-step DSS method is time-consuming to collect sufficient channel data. It is also important to note that some mmWave small-scale channel model parameters are frequency-dependent and environment-specific [22], [23], whereas only a handful of outdoor measurement activities focus on these effects with the same measurement configurations [17], [19].

Previous experimental investigations indicate that a large fraction of signal energy concentrate on the direct path in line-of-sight (LoS) scenarios, and the specular reflection from building exterior walls plays a role in non-LoS (NLoS) scenarios [13], [14]. These observations are also in line with the results in [24], where outdoor building materials are excellent reflectors with large reflection coefficients, but ground reflection is negligible [25]. The analysis of diffraction loss over rooftop and building corners has been provided in [26]-[28] based on knife-edge diffraction (KED) model and creeping wave linear model, which are derived as a function of diffraction angle. However, little is known about the implications of these outdoor mmWave propagation mechanisms on system design with extending coverage.

In this work, we remedy these gaps based on a comprehensive measurement campaign conducted in two typical urban macrocell (UMa) scenarios across $28 \mathrm{GHz}$ and $39 \mathrm{GHz}$ using our custom-designed high-efficiency channel sounder [29]. The contributions of this work are as follows.

1) The details of cellular-type channel measurement campaigns performed in urban central business district (CBD) and dense residential area (DRA) environments for both LoS and NLoS links at two identified $5 \mathrm{G}$ mmWave bands are presented. Thousands of effective directional power delay profiles (PDPs) are collected for statistical channel modeling.

2) An improved auto-clustering algorithm is developed, including initial cluster centroid s- 
election, two-step KPowerMeans clustering, outliers detection and pruning, and cluster validation, which takes account of sparse structure of mmWave channels in delay and angular domains. Its advantages in such aspects as clustering accuracy and efficiency could distinguish temporally sparse clusters with lower number of iteration.

3) The cluster-level parameters are estimated, including the Ricean K-factor, number of clusters, delay spread (DS), and azimuth and zenith angular spreads of arrival (ASA/ZSA). We compare the distributions and statistics of these channel parameters across multiple carrier frequency and LOS/NLOS links and observe weak correlations among them. The impact of different propagation mechanisms on the birth of clusters in several NLoS scenarios is investigated. Modeling results complete present 3GPP channel models above $6 \mathrm{GHz}$ [23].

The rest of this paper is organized as follows. Section II describes the channel sounder hardware and measurement environments. Cluster-based channel characterization of two UMa scenarios for both LoS and NLoS links are presented in Section III and IV, including clusterlevel channel parameters and their correlations, as well the impacts of reflection and diffraction. Finally, Section V draws the conclusions.

\section{Cellular-Type Channel Measurements}

\section{A. Channel Sounder Hardware and Setup}

A brief description of the custom-designed mmWave channel sounder using commercial offthe-shelf (COTS) instruments is given here, and more details can be found in [29]. Fig. 1 depicts the schematic of the channel measurement system. At the transmitter (TX) side, a highperformance vector signal generator (VSG) is used to continuously transmit a binary periodic complementary Golay pair of length 4096 stated at the clock rate of $300 \mathrm{MHz}$. The signals of radio frequency $(\mathrm{RF})$ null-to-null bandwidth of $600 \mathrm{MHz}$ at center frequencies of $28 \mathrm{GHz}$ and $39 \mathrm{GHz}$, corresponding to the delay resolution of $3.3 \mathrm{~ns}$, are emitted via a wideband wide-beam horn antenna fixed on a tripod. Compared with the widely used pseudonoise (PN) sequence, this 


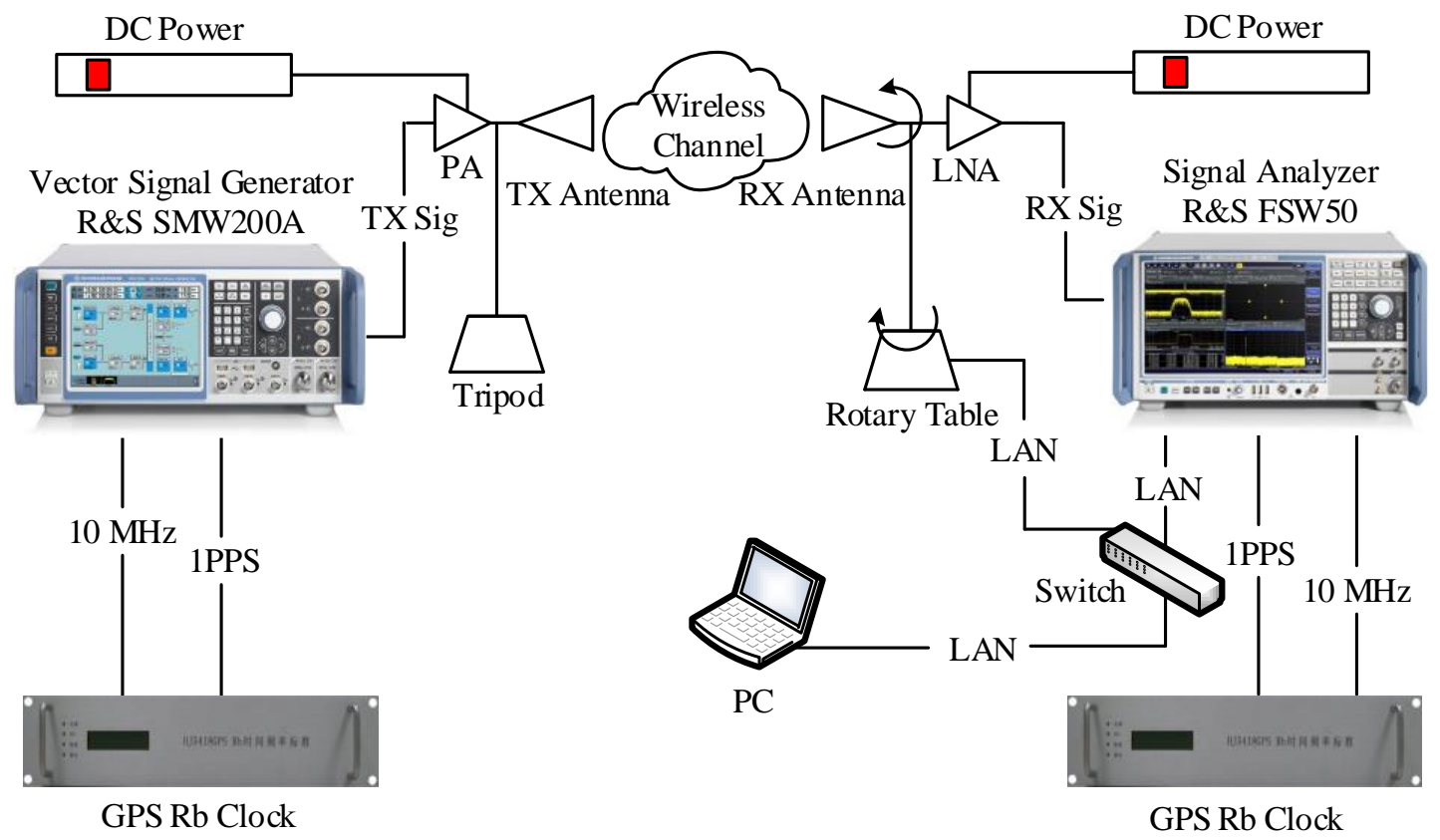

Fig. 1. The COTS instruments based mmWave channel measurement setup description in dense urban environments.

Golay pair exhibits perfect complementary auto-correlation properties [30]. At the receiver (RX) side, a vector signal analyzer (VSA) is utilized for raw data acquisition. The narrow beam horn antennas are placed on a custom-designed positioner to scan in azimuth and elevation directions automatically, enabling the capturing of spatial multipath signals. Both the transceiver antennas are vertically polarized. In addition, power amplifiers (PAs) and low noise amplifiers (LNAs) for each concerned band are used to extend system's dynamic range.

Table I reports the measurement settings for the sounder at $28 \mathrm{GHz}$ and $39 \mathrm{GHz}$. Note that the specifications for TX and RX antennas, such as half-power beamwidth (HPBW) and antenna gain, are obtained based on measurement results. The overall measurable path loss of the sounder, i.e., $154 \mathrm{~dB}$ and $159 \mathrm{~dB}$ at $28 \mathrm{GHz}$ and $39 \mathrm{GHz}$, respectively, is high enough to collect sufficient sounding data with long propagation distance for statistical channel modeling. As for computing the dynamic range of the sounder (i.e., the RX) [21], the estimated received power is beyond the RX sensitivity when the input power to the RX RF connector is $-71 \mathrm{dBm}$ and $-73 \mathrm{dBm}$ at 
TABLE I

Specifications of Broadband Multi-Frequency Channel Sounder

\begin{tabular}{l|c|c}
\hline Parameter & \multicolumn{2}{|c}{ Value } \\
\hline \hline Carrier frequency & $28 \mathrm{GHz}$ & $39 \mathrm{GHz}$ \\
\hline Clock rate of the sequence & \multicolumn{2}{|c}{$300 \mathrm{MHz}$} \\
\hline RF null-to-null bandwidth & \multicolumn{2}{|c}{$600 \mathrm{MHz}$} \\
\hline Delay resolution & \multicolumn{2}{|c}{$3.3 \mathrm{~ns}$} \\
\hline TX antenna HPBW & $60^{\circ}$ & $50^{\circ}$ \\
\hline TX antenna gain & $11.4 \mathrm{~dB}$ & $18.7 \mathrm{~dB}$ \\
\hline PA gain & $20 \mathrm{~dB}$ & $18 \mathrm{~dB}$ \\
\hline PA P1dB & $30 \mathrm{dBm}$ & $28 \mathrm{dBm}$ \\
\hline RX antenna HPBW & $9.5^{\circ}$ & $9.5^{\circ}$ \\
\hline RX antenna gain & $25.6 \mathrm{~dB}$ & $27.7 \mathrm{~dB}$ \\
\hline LNA gain & $33 \mathrm{~dB}$ & $35 \mathrm{~dB}$ \\
\hline Polarization & Vertical-Vertical \\
\hline
\end{tabular}

$28 \mathrm{GHz}$ and $39 \mathrm{GHz}$, respectively. The field measured 1-dB compression input power for the two LNAs is respectively $-16 \mathrm{dBm}$ and $-21 \mathrm{dBm}$ at $28 \mathrm{GHz}$ and $39 \mathrm{GHz}$, so the dynamic range of the sounder is $55 \mathrm{~dB}$ and $52 \mathrm{~dB}$ in these two bands.

Here, two separate GPS Rubidium $(\mathrm{Rb})$ standard references are connected to the VSG and the VSA, respectively, for frequency synchronization with stable $10 \mathrm{MHz}$ outputs and data reception with periodical 1 pulse-per-second (1PPS) trigger signals. Thanks to the employment of $\mathrm{Rb}$ standard references, absolute propagation delay can be obtained following the calibration method developed in [31]. However, only relative propagation delay was considered in this work due to the measurement limitations in actual outdoor environments. During the measurements, an uninterruptible power supply (UPS) unit was used at the RX cart with up to 4 hours of battery power.

The back-to-back calibrations are performed before the measurements via directly connecting 


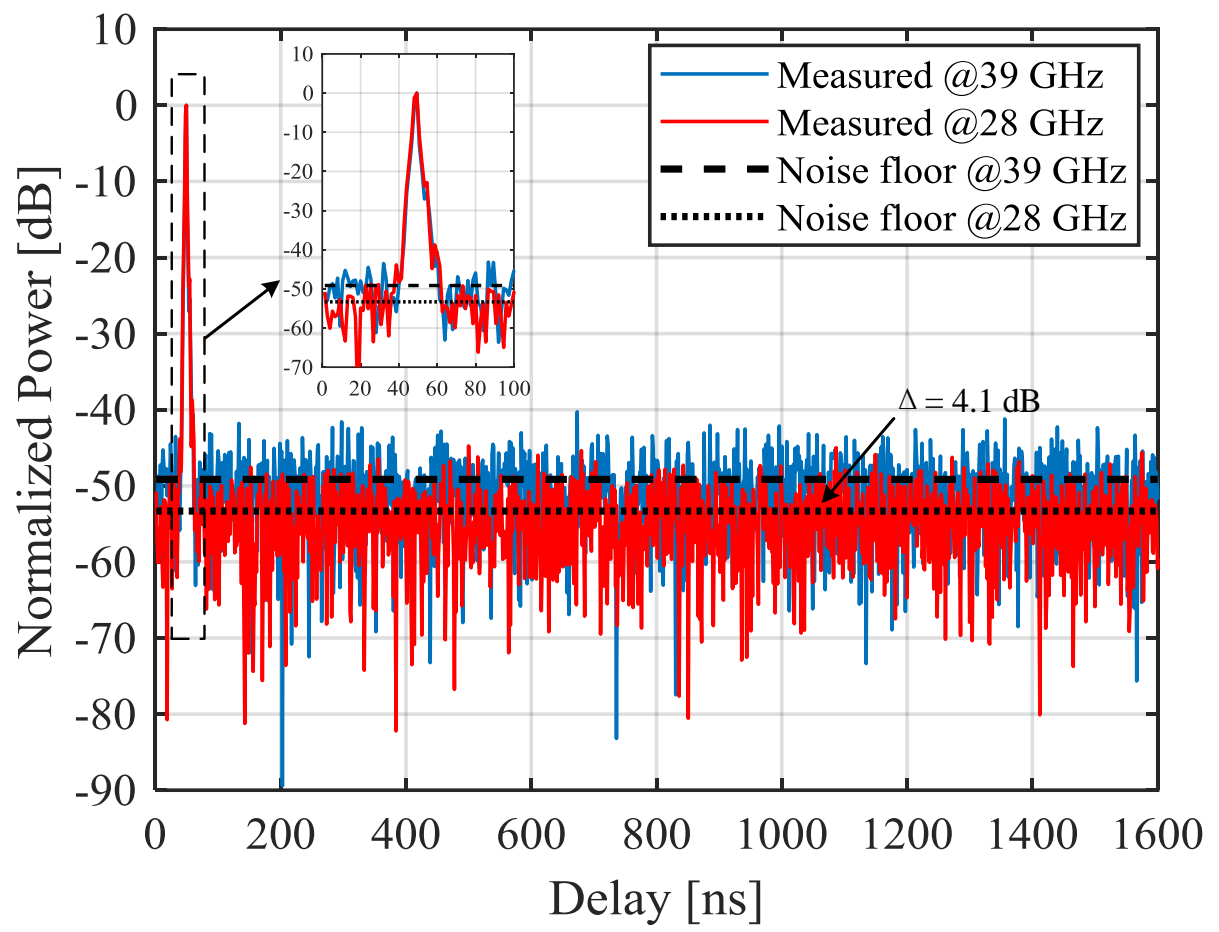

Fig. 2. Normalized PDPs for the system responses at $28 \mathrm{GHz}$ and $39 \mathrm{GHz}$ obtained via back-to-back measurements with transmitted power of $-65 \mathrm{dBm}$ including the impact of the PA at the transmitter and the LNA at the receiver.

transceiver RF front ends via a fixed attenuator in which the PA at the transmitter and the LNA at the received are included. Fig. 2 shows the normalized PDP for the thru connection in the back-to-back tests at $28 \mathrm{GHz}$ and $39 \mathrm{GHz}$ with the transmitted power of $-65 \mathrm{dBm}$. During the calibration measurements, the sounding sequence is transmitted 10 times for average in the two considered bands. Thanks to using the COTS instruments, it shows better temporal stability of calibrations. Notice that the normalized noise floor at $28 \mathrm{GHz}$ is $4.1 \mathrm{~dB}$ lower than that at $39 \mathrm{GHz}$. The captured system impulse responses ensure a precise PDP estimation after deembedding the impact of channel sounder and can be utilized to calibrate the insertion loss and the transmission delay of the COTS-based sounder. Here, the threshold of $8 \mathrm{~dB}$ above the noise floor is employed, resulting in $37.9 \mathrm{~dB}$ and $33.3 \mathrm{~dB}$ system gains at $28 \mathrm{GHz}$ and $39 \mathrm{GHz}$, respectively. 
TABLE II

TX-RX LOCATion COMbinations AND THEIR SERVING SECTORS

\begin{tabular}{c|c|c|c|c}
\hline Envi. & Sector (Azi. Angle) & Scenario & RX Position & Valid \\
\hline \hline \multirow{3}{*}{ CBD } & Sector $1\left(-20^{\circ}\right)$ & $\operatorname{LoS}$ & $1-18$ & $1-13$ \\
\cline { 2 - 5 } & Sector 2 $\left(+40^{\circ}\right)$ & $\operatorname{LoS}$ & $12-25$ & $14-25$ \\
& & NLoS & $26-46$ & $26-43$ \\
\hline \multirow{3}{*}{ DRA } & Sector $1\left(-20^{\circ}\right)$ & $\operatorname{LoS}$ & $4-11,16,22-24$ & $4-11,16,22-24$ \\
\cline { 2 - 5 } & & NLoS & $1-3,12-15,17-21$ & $1-3,12-15,17-21$ \\
\cline { 2 - 5 } & Sector 2 $\left(+40^{\circ}\right)$ & $\operatorname{LoS}$ & $22-24$ & $/$ \\
& & NLoS & $25-36$ & $25-35$ \\
\hline
\end{tabular}

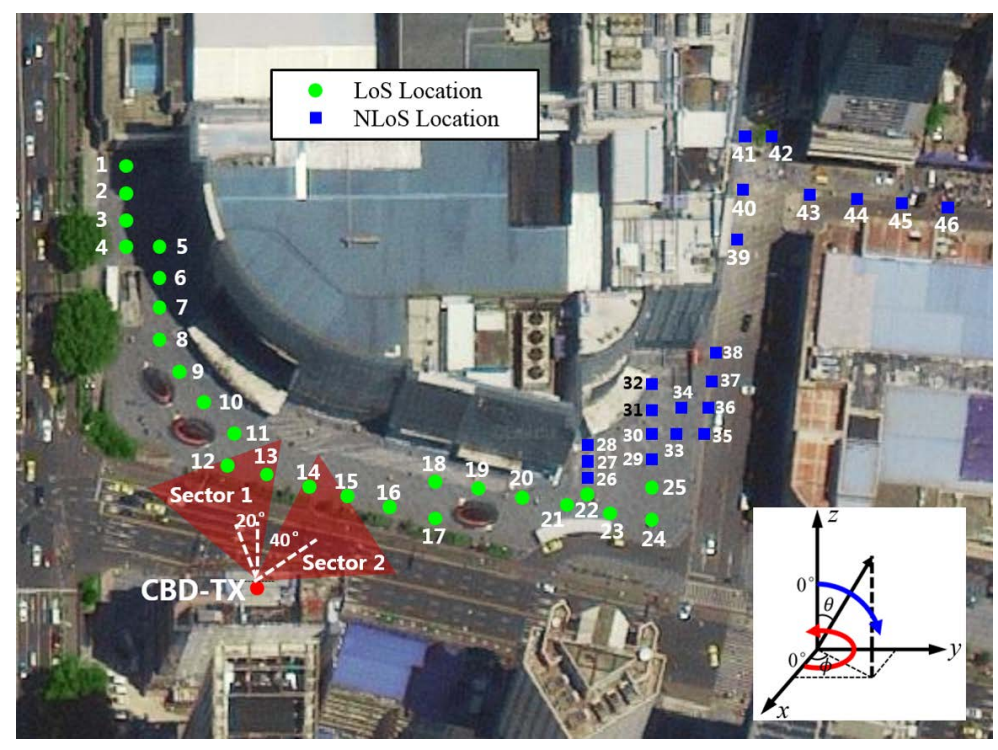

(a)

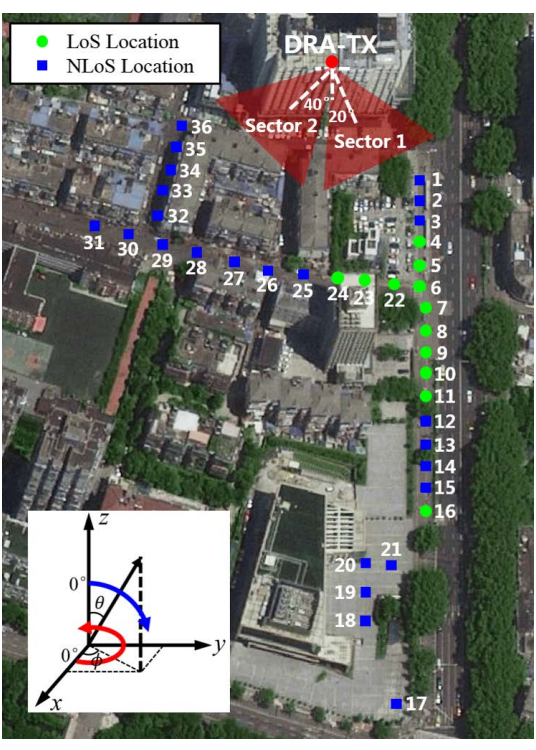

(b)

Fig. 3. TX and RX locations in (a) CBD and (b) DRA environments, where red dots represent TX locations on the rooftop of buildings, green dots and blue squares represent RX locations in LoS and NLoS scenarios, respectively.

\section{B. Measurement Environments and Procedures}

As shown in Fig. 3, outdoor macrocell channel measurements were conducted at both 28 $\mathrm{GHz}$ and $39 \mathrm{GHz}$ in the downtown of Nanjing, China, which provides two typical dense urban environments. Note that the measurement campaigns were both conducted from 11 PM to 
5 AM such that channels were generally wide-sense stationary for very few moving objects (e.g., pedestrians and vehicles). The first set of UMa measurements were obtained in the CBD environment as shown in Fig. 3(a), where the surrounding buildings were coated with metal billboards or LED screens. The CBD-TX was located on the rooftop of the Hanting Hotel (14story high, $45 \mathrm{~m}$ above ground level). Overall, $46 \mathrm{RX}$ locations (1.9 $\mathrm{m}$ height) were chosen, including 25 locations in LoS scenario served by 2 sectors and 21 locations in NLoS scenario only served by Sector $2^{1}$. The TX-RX separation distances were between 56 and $202 \mathrm{~m}$. The second set of UMa measurements were obtained in the DRA environment as shown in Fig. 3(b). The distinguishing feature of this environment is the concrete walls found on the building exteriors leading to poor reflections compared with CBD environment. Measurement data was collected with the DRA-TX located on the rooftop of Sumao Building at a height of $50 \mathrm{~m}$ above ground, while the RX was at $1.9 \mathrm{~m}$ and moved to 36 locations with TX-RX link distances ranging from 55 to $280 \mathrm{~m}$. Among all the $36 \mathrm{RX}$ locations, $13 \mathrm{LoS}$ locations and $23 \mathrm{NLoS}$ locations were taken into consideration. Note that NLoS links in DRA represent no clear direct path existing due to the blockage by large tree trunks (e.g., RX 12-15) or buildings (e.g., RX 17-21 and 25-36), whereas in CBD, all NLoS RXs were blocked by the surrounding buildings. For convenience, Table II provides the TX-RX location combinations and their corresponding sectors in operation.

The PDP measurements in the two dense urban environments are taken for two specific TX azimuth angles, $-20^{\circ}$ and $+40^{\circ}$, using a fixed TX antenna downtilt of $105^{\circ}$ for sector coverage. Then, five different RX elevations of $-70^{\circ},-80^{\circ}, 90^{\circ},+100^{\circ}$, and $+110^{\circ}$ are used, and the whole $360^{\circ}$ azimuthal scans of the RX antennas are conducted in increments of $10^{\circ}$.

\footnotetext{
${ }^{1}$ LoS and NLoS locations are classified according to whether TX and RX horn antennas can align perfectly on boresight via a laser rangefinder.
} 


\section{Cluster-Based Channel Characterization}

\section{A. Clustering Multipath Components}

Effective multipath components (MPCs) and their characterizations, including power $p$, delay $\tau$, azimuth angle of arrival (AoA) $\phi$, and $\operatorname{ZoA} \theta$, are the basic input of clustering algorithm, which can be extracted from directional PDP using peak detection algorithm. If the peak power is larger than the detection level $P_{\mathrm{D}}$, this peak will be selected as an effective MPC. The $P_{\mathrm{D}}$ (in $\mathrm{dB})$ is defined as

$$
P_{\mathrm{D}}=\max \left\{P_{\max }-P_{\mathrm{th}}, N_{o}+\gamma\right\}
$$

where $P_{\text {th }}$ is the power threshold relative to the maximum received peak power $P_{\max }, N_{o}$ is the noise floor calculated by the variance of last hundred ns of each channel impulse response (CIR), and $\gamma$ is the signal-to-noise ratio (SNR). The maximum received peak power $P_{\max }$ is computed as

$$
P_{\max }=\max _{1 \leq i \leq 180} p_{\max , i}
$$

where $p_{\max , i}$ denotes the peak power for the $i$ th directional PDP and there are total 180 directional PDPs (36 in azimuth plane and 5 in elevation plane) at each TX-RX pair. Both $P_{\text {th }}$ and SNR are empirical values depending on carrier frequency, bandwidth, and environments, and in general, contain the typical value of $3 \mathrm{~dB}$ as safety margin.

Fig. 4 shows a typical measured directional PDP for the NLoS scenario in the CBD environment, where the effective PDP filtering the contribution of noise floor (red part) is estimated following the method proposed in [32]. The $30 \mathrm{~dB}$ power threshold and $20 \mathrm{~dB}$ SNR are employed to compute $P_{\mathrm{D}}$. It can be observed that two time clusters with different time durations are identified using the similar representation in [33]. Sequentially a total of 7 effective MPCs (the black marks shown by the insets), whose received power levels are above $-76.42 \mathrm{dBm}$ (the green dotted line), are detected directly from the measured data points using peak detection algorithm. These effective MPCs share the same AoA and ZoA identified during the measurements but 


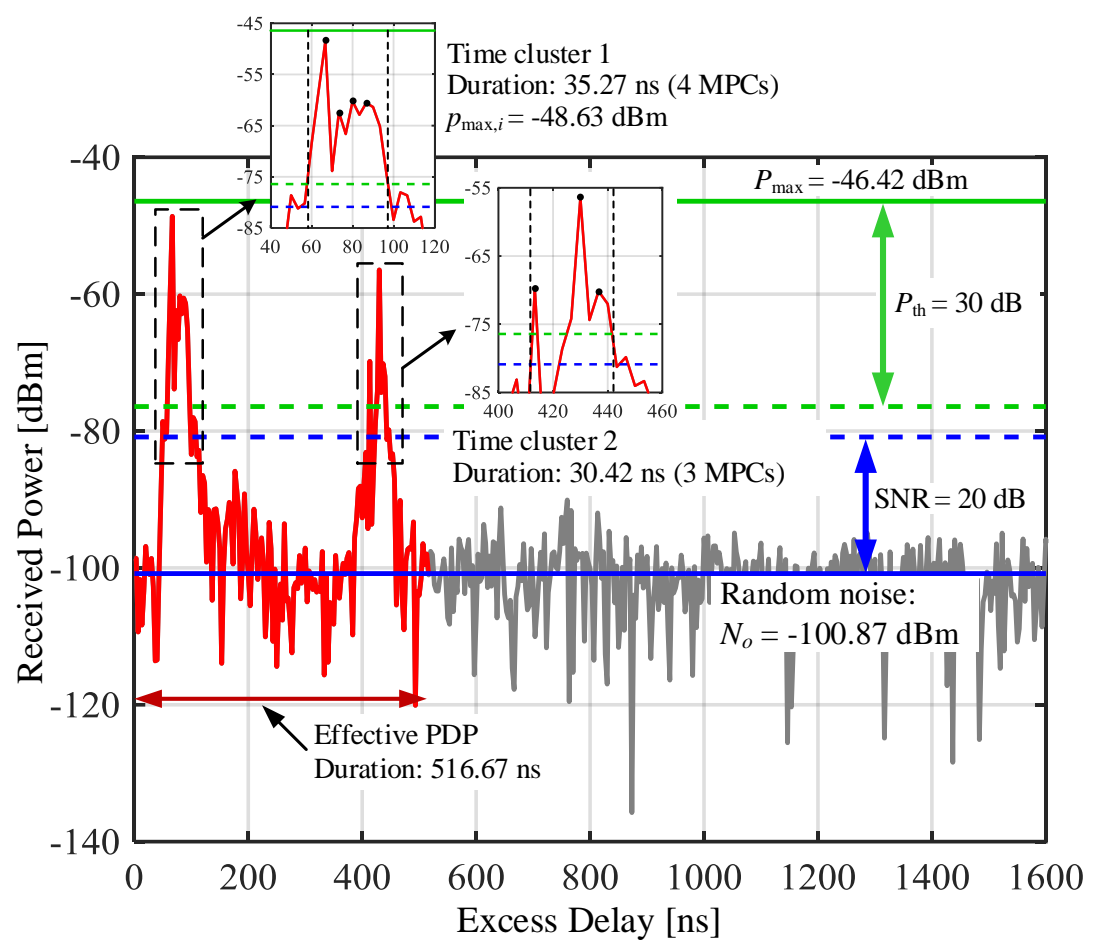

Fig. 4. An illustration of peak detection algorithm to extract effective MPCs based on the typical measured directional PDP at $28 \mathrm{GHz}$ for CBD-RX 38 with $\theta=340^{\circ}$ and $\phi=80^{\circ}$, where two time clusters and a total of 7 MPCs are estimated.

have various propagation delay which mainly concentrate in two time clusters with approximate 363.33 ns difference. Note that the larger $P_{\text {th }}$ or the smaller SNR will result in more weak MPCs to be detected, whereas it has slight impact on clustering results considering outlier detection and pruning procedures in the further developed multipath clustering algorithm.

The cluster nature of mmWave multipath channels indicates that clustering MPCs into several groups is a natural choice to investigate the impact of physical objects on mmWave propagation and exploit low-complexity low-power-consumption transceiver structures with respect to clusterlevel space-time characteristics. The improvements of auto-clustering algorithms require that mmWave propagation characteristics in different environments be taken into account and to use a reasonable similarity measure to quantify the distance between any two individual MPCs with clear physical explanation [34]. 
The widely used MPC distance (MCD) provides a distance measure of two MPCs in both delay and angular domains. Here, the MCD between the $i$ th and the $j$ th $(i, j \in\{1,2, \ldots, L\}$, $i \neq j$ ) MPCs [35] in terms of our single-directional channel measurements is defined as

$$
\mathrm{MCD}_{i j}=\sqrt{\mathrm{MCD}_{\boldsymbol{\Phi}_{i j}}^{2}+\mathrm{MCD}_{\tau_{i j}}^{2}}
$$

where $L$ is the number of estimated MPCs, the angular MCD of arrival is computed as

$$
\operatorname{MCD}_{\boldsymbol{\Phi}_{i j}}=\frac{1}{2}\left\|\boldsymbol{\Phi}_{i}-\boldsymbol{\Phi}_{j}\right\|_{2}
$$

with $\boldsymbol{\Phi}=[\sin \theta \cos \phi, \sin \theta \sin \phi, \cos \theta]^{\mathrm{T}}$, the delay MCD is computed as

$$
\operatorname{MCD}_{\tau_{i j}}=\xi \frac{\left|\tau_{i}-\tau_{j}\right|}{\Delta \tau_{\max }} \frac{\tau_{\text {std }}}{\Delta \tau_{\max }}
$$

with $\xi$ being the delay scaling factor, $\Delta \tau_{\max }$ is the maximum delay difference over all pairs of MPCs, and $\tau_{\text {std }}$ is the standard deviation of delay. However, the delay differences among the finite detectable clusters in outdoor rich scattering environments are up to hundreds of ns (e.g., as shown in Fig. 4, where cluster 2 has approximate 363.33 ns excess delay compared with cluster 1), leading to an imprecise measure of $\Delta \tau_{\max }$ and $\tau_{\text {std }}$ when clustering compact MPCs in time domain.

Hence, an improved auto-clustering algorithm is proposed to cluster MPCs twice, and for each step, the KPowerMeans clustering technique is used, which takes the MPC power into consideration [36]. The KPowerMeans algorithm assigns each MPC to the closest cluster centroid, and updates all the cluster centroids until they no longer change compared with the last iteration. The cluster centroid is defined as a MPC, which has a power-weighted mean of minimum sum of the distance to other MPCs within a cluster. During the clustering, the first step is mainly to distinguish all MPCs in delay domain usually using a large default value of $\xi=10$, and the second step is mainly to group the MPCs in angular domain within each subset using the delay scaling factor $\xi$ computed as [37]

$$
\xi=\frac{\sqrt{\sum_{i=1}^{L_{n}} \sum_{j=1}^{L_{n}} \mathrm{MCD}_{\tau_{i j}}^{2}}}{\sum_{i=1}^{L_{n}} \sum_{j=1}^{L_{n}}\left(\frac{\left|\tau_{i}-\tau_{j}\right|}{\Delta \tau_{\max }} \frac{\tau_{\mathrm{std}}}{\Delta \tau_{\max }}\right)},
$$


where $L_{n}$ is the number of MPCs in the $n$th subset satisfying $\sum_{n=1}^{N} L_{n}=L$. The improved clustering algorithm first clusters the whole MPC set into several small data sets, while a more precise measure of delay MCD in (5) is performed for each small data set in the second-step clustering.

In addition, the KPowerMeans algorithm requires to specific a range for the expected number of clusters $N$ and select initial cluster centroids as inputs. It is worthy to mention that the convergence speed of clustering algorithm mostly depends on the initial centroid selection, where the nearer the initial centroid positions approximates to the final clustering results, the less number of iteration is likely to be required. We thus consider a dissimilarity matrix $\mathbf{S}=\left(s_{i j}\right)_{L \times L}$ with $s_{i j}$ given by

$$
s_{i j}=\frac{p_{i}+p_{j}}{2 P_{\max }} \mathrm{MCD}_{i j}
$$

While a pair of MPCs has as large path power and MCD as possible, corresponding to the increment of $s_{i j}$, these two MPCs can be selected as initial cluster centroids. After clustering $L$ MPCs into final $K$ clusters, where $K$ is usually larger than $N$ due to the second clustering, it is necessary to detect and prune outliers. Here, two kinds of outliers are defined. First, for the cluster with few weak MPCs, we suppose that these MPCs do not belong to any cluster and should be removed directly. Second, some MPCs may stand out from a cluster due to unpredictable channel changing, so an eccentricity $\Delta_{i}$ is used to measure how outliers stand out from the cluster centroid, expressed as

$$
\Delta_{i}=\frac{\mathrm{MCD}_{i, g_{k}}}{\frac{1}{L_{k}} \sum_{j=1}^{L_{k}} \mathrm{MCD}_{j, g_{k}}}
$$

where $g_{k}$ is the index of cluster centroid in the $k$ th cluster with total $L_{k}$ MPCs. If $\Delta_{i}$ is larger than the threshold $\Delta_{\text {th }}$, the $i$ th MPC is considered as an outlier and pruned away. The unitless value of $\Delta_{\text {th }}$ is determined by the rule that $99.7 \%$ of the values $\Delta_{i}$ are within three times of standard deviations. To find the optimal number of cluster $K$, several cluster validation methods 


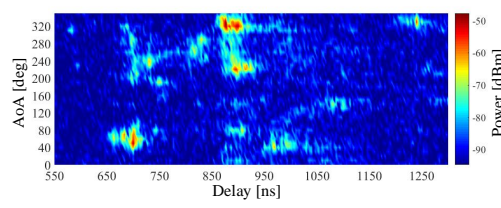

(a)

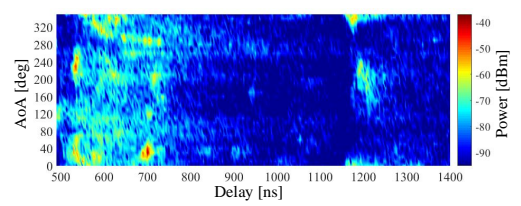

(d)

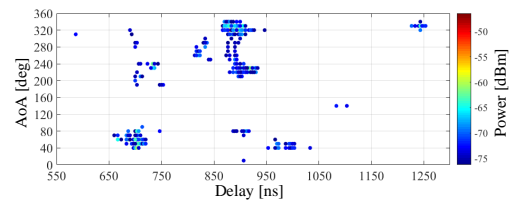

(b)

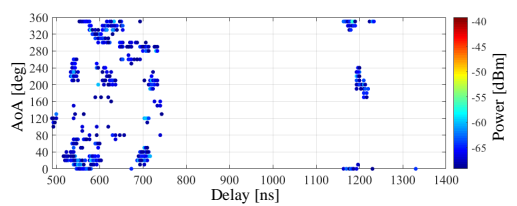

(e)

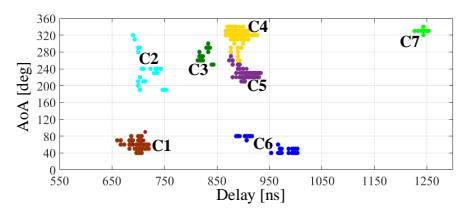

(c)

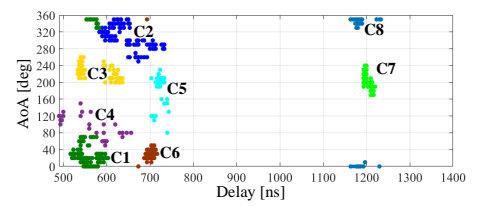

(f)

Fig. 5. Synthesized PDAP calculated from total $180 \mathrm{CIRs}$ with different $(\phi, \theta)$ at $28 \mathrm{GHz}$, and the multipath clustering results based on estimated MPCs. (a), (b), and (c) for the RX 38 in CBD. (d), (e), and (f) for the RX 29 in DRA

can be used, rather than visual inspection based on unpruned clusters [38]. We thus apply the Silhouette index to determine final clustering results with the maximum validation factor [39].

Fig. 5 depicts an example of synthesized power delay angular profile (PDAP) calculated based on 180 measured CIRs for CBD-RX 38 at $28 \mathrm{GHz}$ when RX antenna is rotated in azimuth and elevation, as well as the MPC estimation and clustering results. It can be observed from Fig. 5(a) that received signal energy mainly concentrates on a limited number of space-time blocks, corresponding to different propagation paths and mechanisms. Fig. 5(b) depicts the 360 estimated MPCs using peak detection method with the power threshold of $-76.4 \mathrm{dBm}$ as illustrated in Fig. 4. Following the proposed clustering algorithm, seven clusters are extracted as shown in Fig. 5(c), where the spots with the same color represent the subpaths in a cluster. The visual inspection shows that the result is reasonable and acceptable since the clusters are separated in delay and angular domain, and intra-cluster rays are compact to each cluster centroid after pruning outliers. In combination with the layout-related analysis, cluster $\mathrm{C} 1$ correspond to the singlereflection from the metal billboard on the right side of RX 38. Clusters C2 and C3 correspond to the double-reflection from the nearby and far LED screens on the left side, respectively, 
which exhibit approximately $180^{\circ}$ AoA differences in comparison with cluster C1. Cluster C4 corresponds to the double-reflection (first by the LED screen and then by the external wall of the front building) which experiences longer propagation distance compared with the single-bounce cluster $\mathrm{C} 1$ but has larger path power. To the best of our understanding, this is because the AoAs of these two clusters are totally different corresponding to different scatterers in the realistic environments and the MPCs in cluster C4 are generally from the orientation of the main lobe of TX antenna pattern while from the side lobe for cluster C1. Clusters C5 and C6 are assumed to be high-order reflection of cluster C4. Apart from these dominant clusters, weak cluster with large time delay (e.g., cluster C7) is detected, corresponding to more complicated propagation paths. Meanwhile, note that it is more accurate to distinguish different clusters, as well as their birth-death properties, if narrow beam horn antennas with high gain are employed at the TX side.

Another measurement and clustering results in DRA-NLoS scenario are also depicted in Fig. 5, following the same measurement configurations and analysis methods as above, where a total of 565 MPCs and eight clusters are estimated with $-69.23 \mathrm{dBm}$ detection level. To study the mmWave propagation in more detail, clusters corresponding to the surrounding scatterers are identified. Clusters $\mathrm{C} 1, \mathrm{C} 2, \mathrm{C} 4$, and $\mathrm{C} 6$ correspond to reflection from the exterior wall of a five-story building at the back of RX 29, and cluster C3 corresponds to the diffraction over the rooftop of a six-story building in front of RX 29. Cluster C5 corresponds to the two-time reflected cluster from the front building. Cluster C7 is assumed to be a reflection of cluster C8 through the wall of the building behind RX 29. They have long delays with about $150 \mathrm{~m}$ extra propagation distance compared with most of the clusters. Based on the above illustration, reflection from the building exterior walls and diffraction over the building rooftop levels play a role in coverage enhancement of mmWave systems in UMa NLoS scenarios. Moreover, totally different space-time propagation characteristics can be observed, where the sparse nature of mmWave propagation is visible in CBD environment with several well-separated clusters, but 


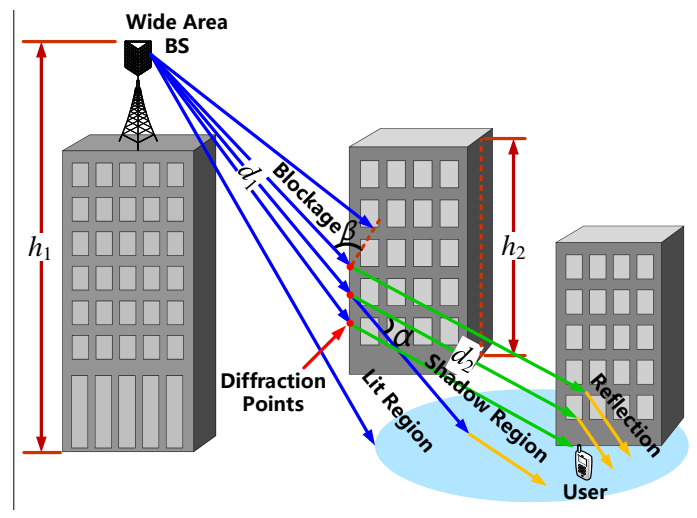

(a)

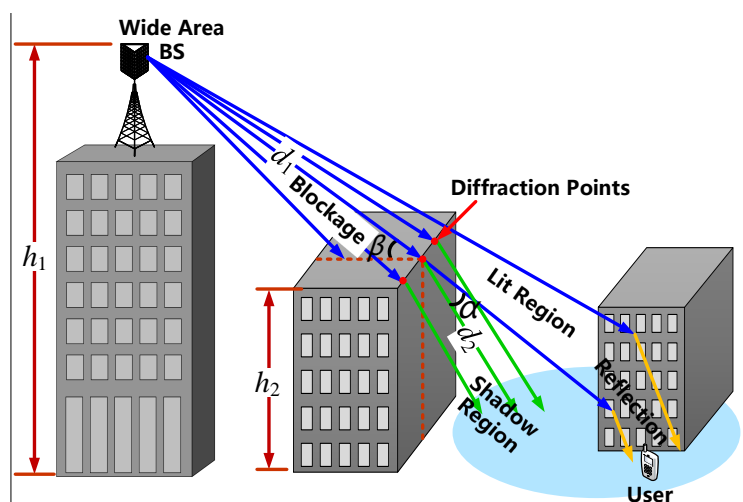

(b)

Fig. 6. Illustration of propagation mechanisms in (a) the CBD-like and (b) the DRA-like NLoS scenarios, where blue lines represent LoS paths, green lines represent diffraction paths, yellow lines represent reflection paths, and red dots represent diffraction points.

the clusters in DRA environment are visually close to each other with probably larger intra-cluster angular spread.

\section{B. Reflection and Diffraction}

As illustrated in Fig. 5, the cluster-level analysis of mmWave propagation characteristics for NLoS links in two dense urban environments show that multiple reflections and diffraction play a role in coverage enhancement of mmWave cellular networks. Existing site-specific channel measurements reveal that reflections become rich at mmWave band due to much shorter wavelength compared with the sizes of surrounding objects [40], [41], whereas there is a dearth of outdoor field measurements to exploit their impacts on channel characterization. On the other hand, two identical horn antennas with narrow HPBW are used for diffraction measurements and always aimed at the diffraction points [26], [27], [42], rather than taking the impact of antenna radiation pattern into account in realistic cellular-type channel modeling. Hence, to exploit optimal transmission scheme for outdoor mmWave cellular system design, the impacts of reflection and diffraction on mmWave space-time propagation characteristics are investigated 
in two typical NLoS cases (see Fig. 6). A diagram of the simplified NLoS case in CBD-like environments is shown in Fig. 6(a), where TX antenna located on the rooftop with height as $h_{1}$, and $h_{2}$ denotes the height of surrounding building which is close to $h_{1}$. Considering a downlink mmWave cellular network, mobile users in shadow region can simultaneously receive diffraction signals over the building corners and reflection signals from lit region when using wide-beam antennas at the TX side. Fig. 6(b) depicts a diagram of the other NLoS case in DRA-like environments, where the height of blockage $h_{2}$ is much lower than TX antenna height $h_{1}$. In general, RX in the shadow region can only receive diffracted signals over the rooftop, and for special case, signals reflected from lit region can also be received to maintain a reliable link when RX is in deep-shadow regions with large diffraction angle. The geometry-based analysis of dominant propagation mechanism reveals that most signals in azimuth plane are blocked by building corner and signals in elevation plane are blocked by building rooftop, leading to huge differences in cluster-level spatial propagation characteristics.

Fig. 7(a) and (b) depict the distribution of received signals in AoA domain varying with RX positions across $28 \mathrm{GHz}$ and $39 \mathrm{GHz}$ in CBD-NLoS scenario, where the number of most effective paths for each TX-RX pair is 30. Combining with the clustering results in power-delayangle domain (e.g., the original received data and clustering result shown in Fig. 5), MPCs with highest power are concentrated within the angular range of $\left[-60^{\circ}, 0^{\circ}\right]$, corresponding to the second-reflection (first by the LED screen on the bottom and then by the external wall of the front building). Meanwhile, remarkable AoA gaps between different reflected clusters indicate that reflection is closely related to the topologies of deployment environments and serves as the dominant propagation mechanism to extend mmWave cell coverage in CBD-like NLoS scenario. Fig. 7(c) and (d) show the measurement results at $28 \mathrm{GHz}$ and $39 \mathrm{GHz}$ in the DRA environment, where the standard deviation of the AoA of the strongest paths over 11 NLoS-RXs is significantly larger than that in the CBD environment. Moreover, it also shows distinct differences between $28 \mathrm{GHz}$ and $39 \mathrm{GHz}$ channels for DRA-RX 27-30, which are blocked by a six-story apartment. 


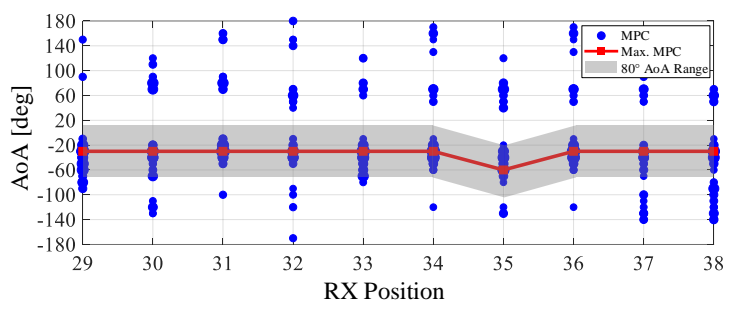

(a)

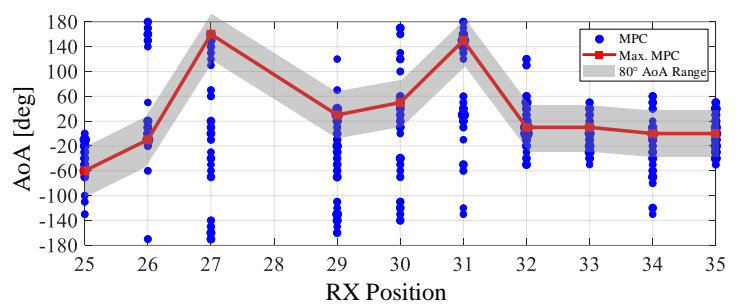

(c)

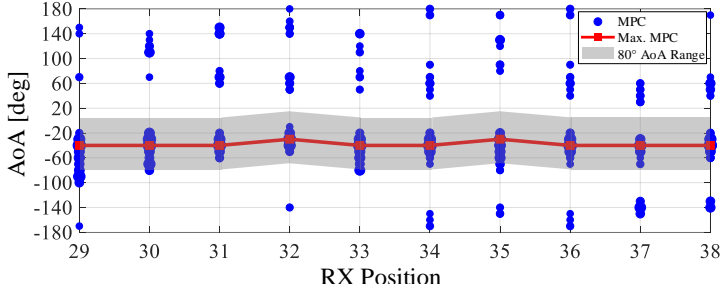

(b)

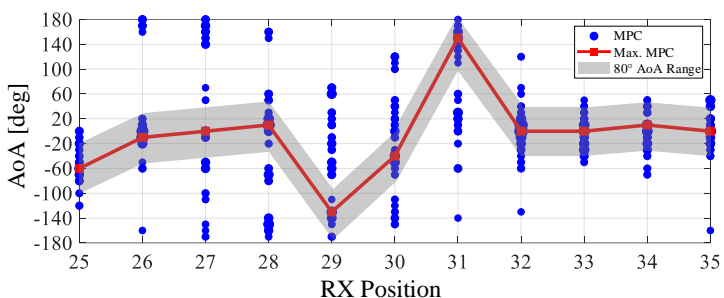

(d)

Fig. 7. The distributions of 30 most effective MPCs for each TX-RX pair with respect to AoA in NLoS scenarios, where red squares represent the MPCs having maximum received power among 30 MPCs and the size of blue dots represent path power. (a) CBD, 28 GHz. (b) CBD, 39 GHz. (c) DRA, 28 GHz. (d) DRA, 39 GHz.

This is due to the fact that the rate of reflection and diffraction is fluctuant, where the diffraction over rooftop level is dominant at $28 \mathrm{GHz}$, and the reflection from exterior wall is prominent at 39 GHz. This trend is in line with the published diffraction measurement results [27], [42], showing that diffraction loss is proportional to carrier frequency, and higher frequencies will cause larger loss at the same diffraction angle. For DRA-RX 25 and 32-35, only clusters formed by specular reflection can be extracted, indicating that extremely large fades need to be overcome in deep shadow regions. The observations from Fig. 7 indicate that cluster-level RMS ASA for CBDNLoS link may smaller than that for DRA-NLoS link since most signals in azimuth plane are blocked by building corners and split into several narrow beamspace to exploit rich reflections from external walls of surrounding buildings. 
TABLE III

Statistics of Cluster-Level Channel Parameters for UMa Scenarios at 28 GHz and 39 GHz Bands

\begin{tabular}{|c|c|c|c|c|c|c|c|c|c|c|c|c|c|}
\hline \multirow{2}{*}{\multicolumn{2}{|c|}{$\begin{array}{c}\text { Environment } \\
\text { Frequency }\end{array}$}} & \multicolumn{4}{|c|}{ UMa, CBD } & \multicolumn{4}{|c|}{ UMa, DRA } & \multicolumn{4}{|c|}{ UMa [23] } \\
\hline & & \multicolumn{2}{|c|}{$28 \mathrm{GHz}$} & \multicolumn{2}{|c|}{$39 \mathrm{GHz}$} & \multicolumn{2}{|c|}{$28 \mathrm{GHz}$} & \multicolumn{2}{|c|}{$39 \mathrm{GHz}$} & \multicolumn{2}{|c|}{$28 \mathrm{GHz}$} & \multicolumn{2}{|c|}{$39 \mathrm{GHz}$} \\
\hline \multicolumn{2}{|l|}{ Scenario } & LoS & NLoS & LoS & NLoS & LoS & NLoS & $\operatorname{LoS}$ & NLoS & LoS & NLoS & $\operatorname{LoS}$ & NLoS \\
\hline \multirow{2}{*}{$\begin{array}{c}\text { Ricean K-factor } \\
\qquad[\mathrm{dB}]\end{array}$} & $\mu$ & 7.34 & NA & 6.27 & NA & 7.71 & NA & 6.37 & NA & 9.00 & NA & 9.00 & NA \\
\hline & $\sigma$ & 2.68 & NA & 2.28 & NA & 1.07 & NA & 2.02 & NA & 3.50 & NA & 3.50 & NA \\
\hline \multirow{2}{*}{ Number of Clusters } & $\mu$ & 7.28 & 5.92 & 7.13 & 5.78 & 6.08 & 6.30 & 5.42 & 5.42 & 12.00 & 20.00 & 12.00 & 20.00 \\
\hline & $\sigma$ & 2.46 & 1.89 & 3.03 & 2.16 & 1.98 & 3.36 & 2.68 & 2.26 & NA & NA & NA & NA \\
\hline \multirow{2}{*}{$\begin{array}{c}\text { Cluster DS } \\
\text { [ns] }\end{array}$} & $\mu$ & 72.13 & 37.37 & 62.81 & 28.30 & 27.00 & 30.56 & 21.09 & 23.09 & 42.63 & 42.63 & 13.78 & 13.78 \\
\hline & $\sigma$ & 41.16 & 31.10 & 46.26 & 29.95 & 23.59 & 27.97 & 24.99 & 23.03 & NA & NA & NA & NA \\
\hline \multirow{2}{*}{$\begin{array}{c}\text { Cluster ASA } \\
{\left[^{\circ}\right]}\end{array}$} & $\mu$ & 14.48 & 10.12 & 12.66 & 8.16 & 13.95 & 14.24 & 11.17 & 13.93 & 11.00 & 15.00 & 11.00 & 15.00 \\
\hline & $\sigma$ & 10.40 & 6.82 & 11.21 & 6.59 & 9.15 & 11.79 & 10.23 & 12.51 & NA & NA & NA & NA \\
\hline \multirow{2}{*}{$\begin{array}{c}\text { Cluster ZSA } \\
{\left[^{\circ}\right]}\end{array}$} & $\mu$ & 7.29 & 6.56 & 7.03 & 8.05 & 9.98 & 7.50 & 7.90 & 7.09 & 7.00 & 7.00 & 7.00 & 7.00 \\
\hline & $\sigma$ & 3.14 & 2.22 & 2.96 & 2.63 & 3.90 & 3.18 & 4.11 & 2.83 & NA & NA & NA & NA \\
\hline
\end{tabular}

\section{Modeling Results And Discussion}

Based on the clustered MPCs for the tens of TX-RX combinations in all scenarios, intercluster and intracluster properties of $28 \mathrm{GHz}$ and $39 \mathrm{GHz}$ propagation channels are analyzed separately.

\section{A. Composite and Intercluster Channel Characteristics}

Table III reports the statistics of intercluster channel parameters including Ricean K-factor and number of clusters $C$, while composite delay and angular dispersions have been reported in [1]. It can be observed that the mean of $C$ reduces with the increment of carrier frequency due to less detectable clusters at higher frequency bands. In CBD environment, the $C$ for NLoS link is significantly smaller than that for LoS link, while there is a slight difference in DRA environment. Moreover, rich scatterers exist in CBD-LoS scenario, resulting in larger $C$ compared with that in DRA-LoS scenario. Unlike the measurement results in this paper, the mean of $C$ was 4.58 for 28 
GHz urban NLoS channel reported in [14], where the DSS method was also used to synthesize omnidirectional channel. The observations in the three dense urban environments indicate that the statistics of $C$ closely related to concerned propagation scenarios and environments. It is also worth noting that the typical numbers of clusters in this work are significantly smaller than the default values for UMa scenario in 3GPP mmWave channel models [23] and WINNER II sub-6 GHz channel model (e.g., 8 and 20 for LOS and NLOS links, respectively). Hence, the channel sparsity in the beamspace domain at mmWave bands should be rather obvious compared with traditional microwave band. With the utilization of large antenna arrays, finite independent clusters indicate that the low rank property of mmWave multiple-input multiple-output (MIMO) channels should be taken into account.

In the case of the LoS condition, the Ricean K-factor is considered as a large-scale channel parameter to scale the power ratio of the dominant MPC (typically the LoS component) and the remaining MPCs [23], [43]. The K-factor is expressed as

$$
K=\frac{V_{\mathrm{D}}^{2}}{2 \sigma^{2}},
$$

where $V_{\mathrm{D}}$ is the amplitude of the main CIR peak (i.e., the LoS component) and $\sigma^{2}$ is the variance of amplitude representing the intensity of the fluctuating part (i.e., the multipath signals). When the K-factor tends to 0 , the channel is dominated by NLoS paths reflected or diffracted from independent physical objects and the channel matrix probably has a large number of singular values. When it tends to $\infty$, the LoS component is dominant for the radio channel with low-rank property. The measured K-factor in CBD and DRA environments are modeled using lognormal distribution, and the goodness of the fit is evaluated by Kolmogorov-Smirnov (K-S) test at $5 \%$ significance level. Their statistics are presented in Table III. It can be seen that the CBD-LoS link has smaller K-factor due to existing rich NLoS MPCs, in comparison with DRA-LoS link at 28 $\mathrm{GHz}$ and $39 \mathrm{GHz}$. The result is consistent with the observations of composite delay and angular spreads reported in [1], where larger K-factor likely leads to less detectable MPCs apart from 


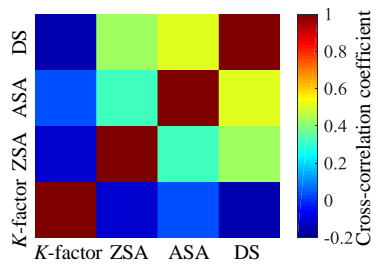

(a)

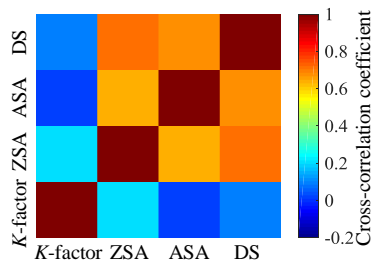

(b)

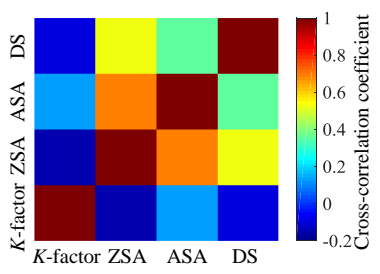

(c)

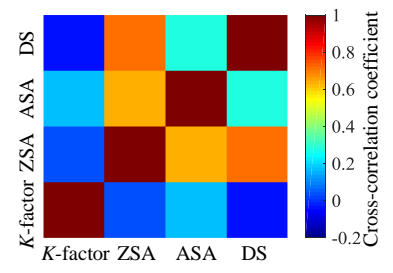

(d)

Fig. 8. Cross-correlation coefficients of Ricean K-factor, composite delay and angular spreads in LoS scenarios. (a) CBD at $28 \mathrm{GHz}$ band. (b) CBD at $39 \mathrm{GHz}$ band. (c) DRA at $28 \mathrm{GHz}$ band. (d) DRA at $39 \mathrm{GHz}$ band.

LoS path, corresponding to smaller composite DS and ASA. Fig. 8 depicts the cross-correlation coefficients of the Ricean K-factor, composite DS, ASA, and ZSA, where correlation coefficients between the Ricean K-factor and RMS dispersion of parameters are generally smaller than zero at $28 \mathrm{GHz}$ for negative correlation, and are within $[-0.2,0.2]$ at $39 \mathrm{GHz}$ for less correlation. In addition, positive correlations among composite DS, ASA, and ZSA can be obtained to predict temporal and angular channel characteristics and improve the reliability of mmWave communication systems.

\section{B. Intracluster Delay and Angular Spreads}

The cluster-level RMS DS is calculated following the similar definition in [44]. Table III identifies the statistics of cluster-based DS (including mean value $\mu$ and standard deviation $\sigma$ ) across different scenarios and frequencies. For LoS scenarios, the average intracluster DS at 28 $\mathrm{GHz}$ and $39 \mathrm{GHz}$ in $\mathrm{CBD}$ are $72.12 \mathrm{~ns}$ and $62.81 \mathrm{~ns}$, respectively, and in DRA are $27.00 \mathrm{~ns}$ and $21.09 \mathrm{~ns}$, respectively. This trend regard to carrier frequency, as expected, depends on the difference in space-time propagation characteristics. Moreover, the mean values of cluster DS in DRA are significantly smaller than those in CBD under identical measurement setup. We speculate that this is because multiple reflections exit in CBD-like rich scattering environment. Note that CBD-LoS scenario has a relatively larger value of $\sigma$ at both $28 \mathrm{GHz}$ and $39 \mathrm{GHz}$, compared with DRA-LoS scenario. This phenomenon is due to the fact that propagation along 
continuous routes with only one sector in operation (e.g., DRA-LoS RXs served by Sector 1) manifests smaller $\sigma$, whereas for CBD-LoS scenario, RXs can be divided into two types (e.g., RX 1-8 along street canyon and RX 9-25 along open square) serving by different sectors.

Fig. 9 depicts the empirical cumulative distribution functions (CDFs) of intracluster DS in the CBD and DRA environments. It can be observed that cluster DS for NLoS links is obviously smaller than that for LoS link in CBD environment, while an opposite result can be observed in DRA environment. A simple explanation for this observation is that more diffused distribution of reflectors can be seen around the RXs in CBD-LoS scenario, leading to significant cluster DS compared with the results in other scenarios. Combining with the analysis for NLoS links in Section III-B, it can be observed that diffraction frequently occurs with smaller propagation delay because it will not significantly increase propagation distance compared with single- and multi-reflections. Moreover, reflection becomes dominant with increasing carrier frequency in DRA-NLoS scenario, corresponding to not that obvious DS differences across two bands in CBD-NLoS scenario. Table III compares the statistics of cluster-level DS obtained from our measurements and 3GPP model [23]. Intuitively, identical cluster DS across LoS and NLoS links are shown in 3GPP model and they are generally smaller than the results derived from this work. Similar behavior can be also observed in comparison with the field measurement result reported in [14], therein mean of cluster DS is 12.86 ns for NLoS link in street canyon at $28 \mathrm{GHz}$. These differences indicate that it is of great importance to consider the effect of propagation environments in the deployment of outdoor mmWave cellular networks.

The parameters associated with the intra-cluster RMS ASA and ZSA across $28 \mathrm{GHz}$ and $39 \mathrm{GHz}$ for all scenarios are also provided in Table III. Fig. 10 shows the CDFs of field measured intra-cluster ASA in CBD and DRA environments. The main conclusions drawn from the observations of the statistics of cluster ASA are the following: 1) cluster-level ASAs are significantly smaller than composite channel parameters, which characterize channel properties of directional transmission in angular domain; 2) an increase in carrier frequency in general 


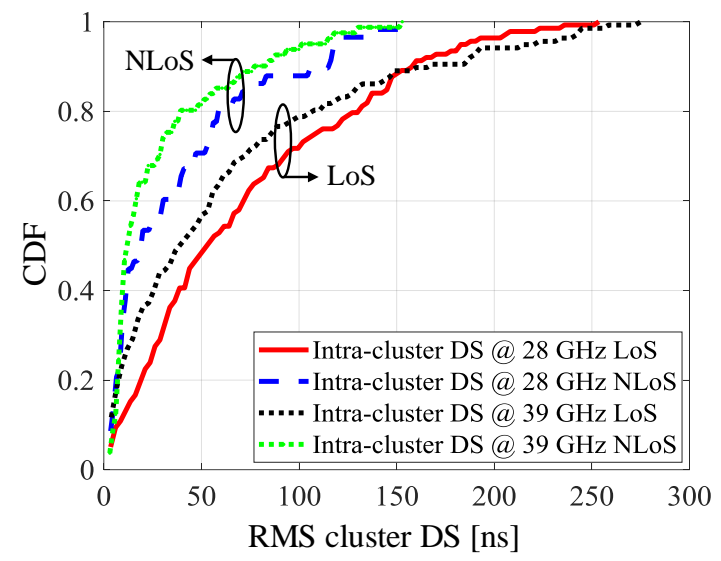

(a)

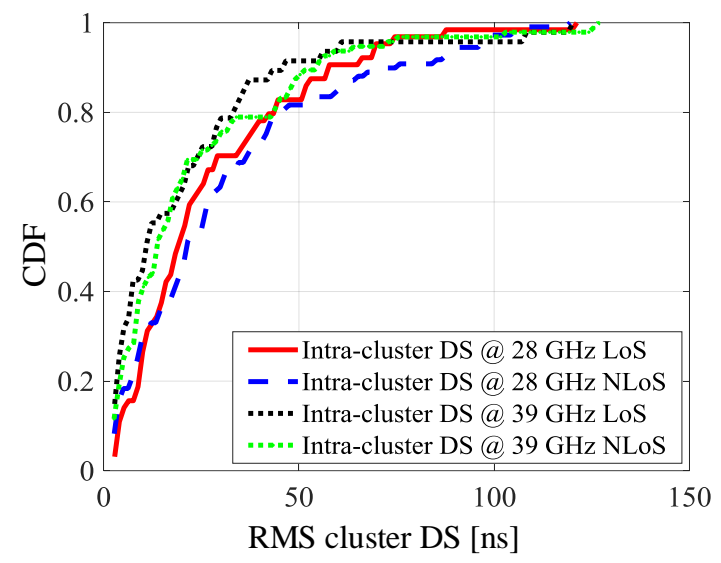

(b)

Fig. 9. Empirical CDF of cluster DS for LoS and NLoS scenarios across $28 \mathrm{GHz}$ and $39 \mathrm{GHz}$ in (a) CBD and (b) DRA environments.

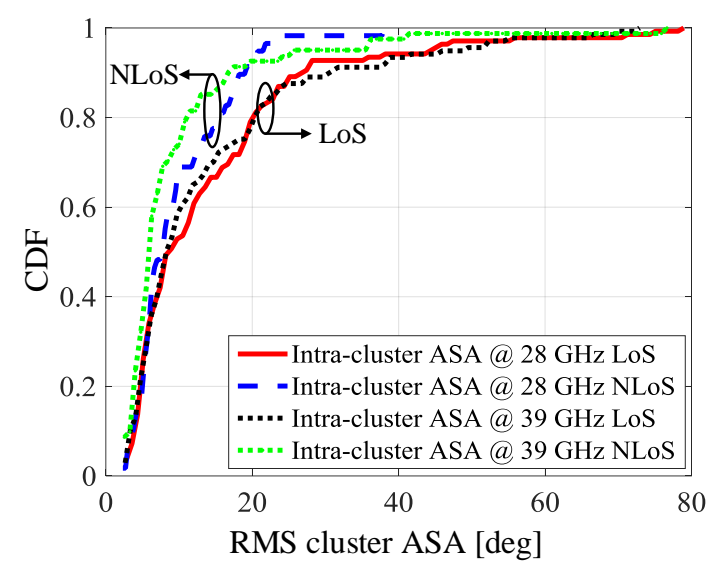

(a)

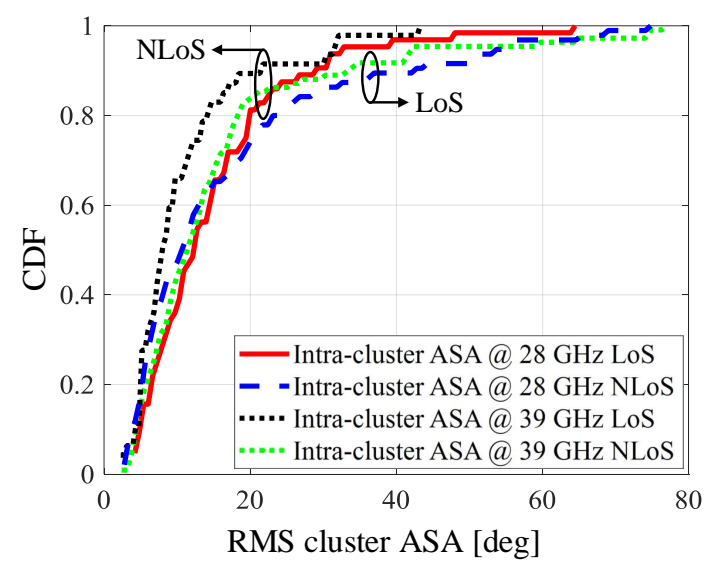

(b)

Fig. 10. Empirical CDF of cluster ASA for LoS and NLoS scenarios across $28 \mathrm{GHz}$ and $39 \mathrm{GHz}$ in (a) CBD and (b) DRA environments.

reduces the RMS cluster ASA for all scenarios, while slight difference can be observed between $28 \mathrm{GHz}$ and $39 \mathrm{GHz} ; 3)$ a similar behavior across LoS and NLoS links is seen between two concerned UMa environments, and this trend is consistent with the results of cluster DS as described before. Also, the means of cluster ZSA are observed to be frequency-independent for all measured scenarios, and the maximum $2.1^{\circ}$ difference between composite and cluster-level RMS 

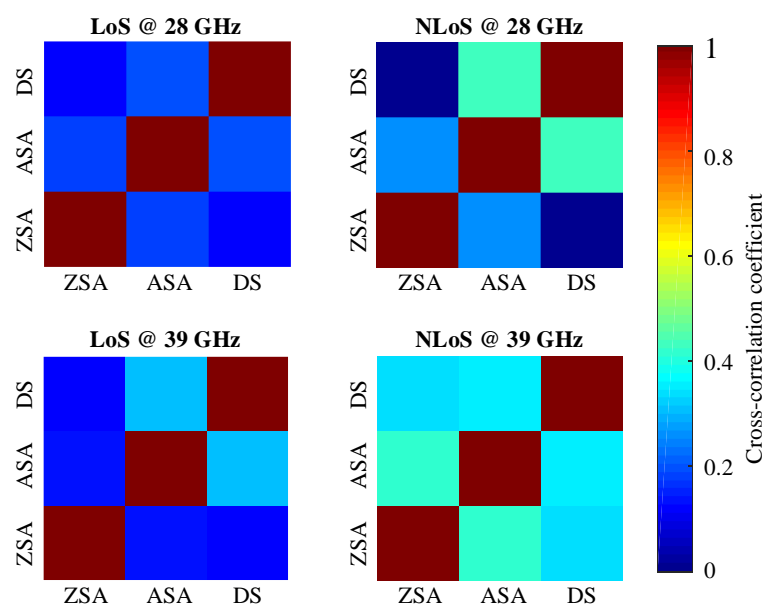

(a)
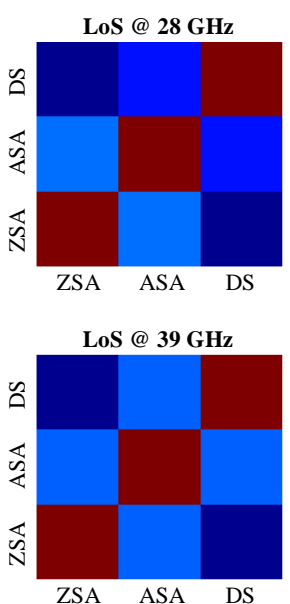

(b)

Fig. 11. Cross-correlation coefficients of cluster-level delay and angular spread in (a) CBD and (b) DRA environments.

ZSA indicates that mmWave propagation in dense urban environments is more concentrated in elevation dimension within the vertical rotation range of $\left[-20^{\circ}, 20^{\circ}\right]$ in our measurements. Hence, low-complexity transmission scheme using two-dimensional (2D) beamforming is feasible in mmWave UMa downlink channels when TX antennas downtilt can be adjusted mechanically and RXs are within the same height.

\section{Correlations of Cluster-Based Channel Parameters}

Fig. 11 depicts the cross-correlation coefficients of cluster-level delay and angular spread across $28 \mathrm{GHz}$ and $39 \mathrm{GHz}$ for both LoS and NLoS links using the same colorbar for convenience. The cross-correlation coefficients among intracluster DS, ASA, and ZSA are generally within [0, 0.2] in LoS scenarios. Intuitively, this means that temporal and spatial propagation characteristics are less correlated or uncorrelated, with respect to the beamspace representation of outdoor mmWave channels with the presence of LoS path. A similar behavior can also be observed for DRA-NLoS link, whereas the cross-correlation coefficients in CBD-NLoS are much larger. This phenomenon is consistent with the trend of intracluster parameters across LoS and NLoS links in CBD and 
DRA environments, due to the fact that well-separated clusters (e.g., as shown in Fig. 5 for CBD-NLoS link) probably share similar intracluster distributions, in comparison to overlapped clusters (e.g., as shown in Fig. 5 for DRA-NLoS link). Hence, the channel vectors are probably not independent and identically distributed in some specific scenarios at mmWave bands, which requires further understanding the impact of actual physical scattering sources, corresponding to different propagation mechanisms, on the space-time variations of the properties of the clusters.

\section{CONClusion}

Based on the extensive radio channel measurement campaigns conducted in CBD and DRA environments across $28 \mathrm{GHz}$ and $39 \mathrm{GHz}$, the cluster-based characterization of outdoor mmWave channels in the UMa scenario has been developed. Thanks to the use of a high-efficiency channel sounder, thousands of directional PDPs with high resolution in delay and angular domains have been collected to identify effective MPCs and clusters using the improved KPowerMeans algorithm.

The mapping results between detected clusters and physical objects indicate that reflection and diffraction are dominant for coverage enhancement and their impacts on channel characteristics mainly depend on the environmental layout. From clustering results, a finite number of strong clusters (e.g., 5-7) would be enough to describe mmWave multipath channels for UMa LoS/NLoS links. Measured composite and intracluster parameters, such as RMS DS and ASA, are all monotonic with frequency, whereas ZSAs are observed to be frequency-independent. A comparison of channel characteristics across LoS and NLoS scenarios shows that in the CBD environment, the statistics of temporal and azimuthal dispersion in the LoS condition are much larger than those in the NLoS condition, while an opposite behavior can be observed in the DRA environment. The cluster-level channel characteristics obtained in this study are more realistic to describe mmWave channels in dense urban environments. 


\section{REFERENCES}

[1] P. Zhang, J. Li, H. Wang, and X. You, "Millimeter-wave space-time propagation characteristics in urban macrocell scenarios," in Proc. IEEE Int. Conf. Commun. (ICC), Shanghai, China, May 2019, pp. 1-6.

[2] T. S. Rappaport et al., "Millimeter wave mobile communications for 5G cellular: It will work!” IEEE Access, vol. 1, pp. 335-349, 2013.

[3] A. Ghosh et al., "Millimeter-wave enhanced local area systems: A high-data-rate approach for future wireless networks," IEEE J. Sel. Areas Commun., vol. 32, no. 6, pp. 1152-1163, June 2014.

[4] T. Baykas et al., "IEEE 802.15.3c: The first IEEE wireless standard for data rates over $1 \mathrm{~Gb} / \mathrm{s}$," IEEE Commun. Mag., vol. 49, no. 7, pp. 114-121, July 2011.

[5] T. Nitsche, C. Cordeiro, A. B. Flores, E. W. Knightly, E. Perahia, and J. C. Widmer, "IEEE 802.11ad: Directional 60 GHz communication for multi-gigabit-per-second Wi-Fi,” IEEE Commun. Mag., vol. 52, no. 12, pp. 132-141, Dec. 2014.

[6] H. Wang, W. Hong, J. Chen, B. Sun, and X. Peng, "IEEE 802.11aj (45GHz): A new very high throughput millimeter-wave WLAN system,” China Commun., vol. 11, no. 6, pp. 51-62, Aug. 2014.

[7] M. Xiao et al., "Millimeter wave communications for future mobile networks," IEEE J. Sel. Areas Commun., vol. 35, no. 9, pp. 1909-1935, Sept. 2017.

[8] F. Boccardi, R. W. Heath, A. Lozano, T. L. Marzetta, and P. Popovski, "Five disruptive technology directions for 5G," IEEE Commun. Mag., vol. 52, no. 2, pp. 74-80, Feb. 2014.

[9] J. G. Andrews, T. Bai, M. N. Kulkarni, A. Alkhateeb, A. K. Gupta, and R. W. Heath, "Modeling and analyzing millimeter wave cellular systems," IEEE Tran. Commun., vol. 65, no. 1, pp. 403-430, Jan. 2017.

[10] T. Bai, A. Alkhateeb, and R. W. Heath, "Coverage and capacity of millimeter-wave cellular networks," IEEE Commun. Mag., vol. 52, no. 9, pp. 70-77, Sept. 2014.

[11] X. Yu, J. Zhang, M. Haenggi, and K. B. Letaief, "Coverage analysis for millimeter wave networks: The impact of directional antenna arrays," IEEE J. Sel. Areas Commun., vol. 35, no. 7, pp. 1498-1512, July 2017.

[12] S. Sun, T. S. Rappaport, M. Shafi, P. Tang, J. Zhang, and P. J. Smith, "Propagation models and performance evaluation for 5G millimeter-wave bands," IEEE Tran. Veh. Technol., vol. 67, no. 9, pp. 8422-8439, Sept. 2018.

[13] S. Hur et al., "Proposal on millimeter-wave channel modeling for 5G cellular system," IEEE J. Sel. Topics Signal Process., vol. 10, no. 3, pp. 454-469, Apr. 2016.

[14] J. Ko et al., "Millimeter-wave channel measurements and analysis for statistical spatial channel model in in-building and urban environments at 28 GHz,’ IEEE Trans. Wireless Commun., vol. 16, no. 9, pp. 5853-5868, Sept. 2017.

[15] F. Sohrabi and W. Yu, "Hybrid digital and analog beamforming design for large-scale antenna arrays," IEEE J. Sel. Topics Signal Process., vol. 10, no. 3, pp. 501-513, Apr. 2016.

[16] V. Raghavan, J. Cezanne, S. Subramanian, A. Sampath, and O. Koymen, "Beamforming tradeoffs for initial UE discovery in millimeter-wave MIMO systems,” IEEE J. Sel. Topics Signal Process., vol. 10, no. 3, pp. 543-559, Apr. 2016. 
[17] T. S. Rappaport, E. Ben-Dor, J. N. Murdock, and Y. Qiao, “38 GHz and $60 \mathrm{GHz}$ angle-dependent propagation for cellular \& peer-to-peer wireless communications," in Proc. IEEE Int. Conf. Commun. (ICC), Ottawa, ON, Canada, June 2012, pp. $4568-4573$.

[18] T. S. Rappaport, G. R. MacCartney, M. K. Samimi, and S. Sun, "Wideband millimeter-wave propagation measurements and channel models for future wireless communication system design,” IEEE Trans. Commun., vol. 63, no. 9, pp. 3029-3056, Sept. 2015.

[19] V. Raghavan, A. Partyka, L. Akhoondzadeh-Asl, M. A. Tassoudji, O. H. Koymen, and J. Sanelli, "Millimeter wave channel measurements and implications for PHY layer design,” IEEE Trans. Antennas Propag., vol. 65, no. 12, pp. 6521-6533, Dec. 2017.

[20] K. Haneda, N. Omaki, T. Imai, L. Raschkowski, M. Peter, and A. Roivainen, "Frequency-agile pathloss models for urban street canyons," IEEE Trans. Antennas Propag., vol. 64, no. 5, pp. 1941-1951, May 2016.

[21] C. U. Bas, V. Kristem, R. Wang, and A. F. Molisch, "Real-time ultra-wideband channel sounder design for 318 GHz," IEEE Trans. Wireless Commun., vol. 67, no. 4, pp. 2995-3008, Apr. 2019.

[22] P. Zhang, J. Li, H. Wang, H. Wang, and W. Hong, "Indoor small-scale spatiotemporal propagation characteristics at multiple millimeter-wave bands," IEEE Antennas Wireless Propag. Lett., vol. 17, no. 12, pp. 2250-2254, Dec. 2018.

[23] "Study on channel model for frequency from 0.5 to $100 \mathrm{GHz}$," 3GPP, Tech. Rep. 38.901 (V15.0.0), June 2018.

[24] H. Zhao et al., "28 GHz millimeter wave cellular communication measurements for reflection and penetration loss in and around buildings in New York city," in Proc. IEEE Int. Conf. Commun. (ICC), Budapest, Hungary, June 2013, pp. $5163-5167$.

[25] T. S. Rappaport and S. Deng, "73 GHz wideband millimeter-wave foliage and ground reflection measurements and models," in Proc. IEEE Int. Conf. Commun. Workshop (ICCW), London, UK, June 2015, pp. 1238-1243.

[26] K. Kim, M. Kim, J. Park, J. Lee, J. Liang, and K. Lee, "Diffraction loss model based on 28 GHz over-rooftop propagation measurements," in Proc. IEEE 86th Veh. Technol. Conf. (VTC-Fall), Toronto, ON, Canada, Sept. 2017, pp. 1-5.

[27] T. S. Rappaport, G. R. MacCartney, S. Sun, H. Yan, and S. Deng, "Small-scale, local area, and transitional millimeter wave propagation for 5G communications," IEEE Trans. Antennas Propag., vol. 65, no. 12, pp. 6474-6490, Dec. 2017.

[28] V. Raghavan et al., "Statistical blockage modeling and robustness of beamforming in millimeter-wave systems," IEEE Trans. Microw. Theory Tech., pp. 1-15, 2019.

[29] J. Li, P. Zhang, H. Wang, C. Yu, and W. Hong, "High-efficiency millimeter-wave wideband channel measurement system," in Proc. Eur. Conf. Antennas Propag. (EuCAP), Krakow, Poland, Apr. 2019, pp. 1-5.

[30] H. Wang, X. Gao, B. Jiang, X. You, and W. Hong, "Efficient MIMO channel estimation using complementary sequences," IET Commun., vol. 1, no. 5, pp. 962-969, Oct. 2007.

[31] G. R. MacCartney and T. S. Rappaport, "A flexible millimeter-wave channel sounder with absolute timing," IEEE J. Sel. Areas Commun., vol. 35, no. 6, pp. 1402-1418, June 2017. 
[32] H. Wang, P. Zhang, J. Li, and X. You, "Radio propagation and wireless coverage of LSAA-based 5G millimeter-wave mobile communication systems," China Commun., vol. 16, no. 5, pp. 1-18, May 2019.

[33] M. K. Samimi and T. S. Rappaport, “3-D millimeter-wave statistical channel model for 5G wireless system design,” IEEE Trans. Microw. Theory Tech., vol. 64, no. 7, pp. 2207-2225, July 2016.

[34] R. He et al., "Clustering enabled wireless channel modeling using big data algorithms," IEEE Commun. Mag., vol. 56, no. 5, pp. 177-183, May 2018.

[35] N. Czink, P. Cera, J. Salo, E. Bonek, J.-P. Nuutinen, and J. Ylitalo, "Improving clustering performance using multipath component distance," Electron. Lett., vol. 42, no. 1, pp. 33-45, Jan. 2006.

[36] — - "A framework for automatic clustering of parametric MIMO channel data including path powers," in Proc. IEEE 64th Veh. Technol. Conf. (VTC-Fall), Montreal, Que., Canada, Sept. 2006, pp. 1-5.

[37] C. Ling et al., "Double-directional dual-polarimetric cluster-based characterization of 70-77 GHz indoor channels," IEEE Trans. Antennas Propag., vol. 66, no. 2, pp. 857-870, Feb. 2018.

[38] S. Sangodoyin, V. Kristem, A. F. Molisch, R. He, F. Tufvesson, and H. M. Behairy, "Statistical modeling of ultrawideband MIMO propagation channel in a warehouse environment," IEEE Trans. Antennas Propag., vol. 64, no. 9, pp. 4049-4063, Sept. 2016.

[39] P. Zhang, H. Wang, Y. Zhou, X. Sun, and H. Wang, “An improved three-dimensional auto-clustering algorithm for indoor millimeter-wave multipath propagation analysis," in Proc. IEEE/CIC Int. Conf. Commun. China (ICCC), Qingdao, China, Oct. 2017, pp. 1-6.

[40] K. Sato et al., "Measurements of reflection and transmission characteristics of interior structures of office building in the 60-GHz band," IEEE Trans. Antennas Propag., vol. 45, no. 12, pp. 1783-1792, Dec. 1997.

[41] R. Sun et al., "Millimeter-wave radio channels vs. synthetic beamwidth,” IEEE Commun. Mag., vol. 56, no. 12, pp. 53-59, Dec. 2018.

[42] M. Jacob, S. Priebe, R. Dickhoff, T. Kleine-Ostmann, T. Schrader, and T. Kurner, "Diffraction in mm and sub-mm wave indoor propagation channels," IEEE Trans. Microw. Theory Techn., vol. 60, no. 3, pp. 833-844, Mar. 2012.

[43] C. Huang, A. F. Molisch, R. Wang, P. Tang, R. He, and Z. Zhong, "Angular information-based NLOS/LOS identification for vehicle to vehicle MIMO system," in Proc. IEEE Int. Conf. Commun. Workshops (ICCW), Shanghai, China, May 2019, pp. 1-6.

[44] T. S. Rappaport, R. W. Heath, R. C. Daniels, and J. Murdock, Millimeter Wave Wireless Communications. Englewood Cliffs, NJ, USA: Prentice-Hall, Sept. 2014. 